\title{
ON THE IMAGE AND FIBRES OF SOLVABLE BASE CHANGE
}

\author{
C.S. RAJAN
}

Abstract. Our aim is to describe the image and fibres of the base change lift constructed by Langlands, for solvable extensions of number fields.

\section{Introduction}

A particular instance of the principle of functoriality enunciated by Langlands, is the base change map relating automorphic forms on $G L_{2}\left(\mathbf{A}_{k}\right)$ to forms on $G L_{2}\left(\mathbf{A}_{K}\right)$, for an extension $K / k$ of number fields. Using the trace formula and following earlier work by Saito and Shintani, Langlands in [L], established the existence of the base change lift $B C_{K / k}$ sending cuspidal automorphic representations of $G L_{2}\left(\mathbf{A}_{k}\right)$ to automorphic representations of $G L_{2}\left(\mathbf{A}_{K}\right)$, provided $K / k$ is a cyclic extension of prime degree. Langlands further characterized the image and the fibres of the base change map $B C_{K / k}$.

Given an automorphism $\sigma$ of $K$, and an automorphic representation $\Pi$ of $G L_{2}\left(\mathbf{A}_{K}\right)$, let $\Pi^{\sigma}$ denote the automorphic representation of $G L_{2}\left(\mathbf{A}_{K}\right)$ defined by

$$
\Pi^{\sigma}(g)=\Pi\left(\sigma^{-1}(g)\right) .
$$

The descent property for invariant, automorphic representations established by Langlands is that if $\Pi$ is a cuspidal automorphic representation of $G L_{2}\left(\mathbf{A}_{K}\right)$, which is invariant with respect to the action of the Galois group $G(K / k)$ of a cyclic extension $K / k$ of prime degree, then $\Pi$ lies in the image of the base change map $B C_{K / k}$ from automorphic representations on $G L_{2}\left(\mathbf{A}_{k}\right)$ to automorphic representations on $G L_{2}\left(\mathbf{A}_{K}\right)$. Further if $\pi_{1}$ and $\pi_{2}$ are distinct, cuspidal automorphic representations of $G L_{n}\left(\mathbf{A}_{k}\right)$ which base change to $\Pi$, then

$$
\pi_{1} \simeq \pi_{2} \otimes \chi_{K / k}
$$

where $\chi_{K / k}$ is an idele class character of $k$ which corresponds to the cyclic extension $K / k$ by class field theory. The descent criterion, that invariant automorphic representations descend for cyclic extensions of prime degree, was then utilised by Langlands and the results completed by Tunell, to obtain nonabelian reciprocity laws, in that a strong form of Artin's conjecture for any Galois representation

Received April 29, 2002.

2000 Mathematics Subject Classification. Primary 11F70; Secondary 22E55. 
of the absolute Galois group $G_{K}$ of a number field $K$ to $G L_{2}(\mathbb{C})$ with solvable image was obtained.

The base change map constructed by Langlands, for cyclic extensions of prime degree, allows one to construct inductively the base change map for a solvable extension $K / k$ of number fields. It is natural to ask for a characterisation of the image of the base change lift, in particular whether invariant forms descend, and to know the fibres of the lift.

The descent property for invariant representations with respect to a cyclic group of automorphisms, was obtained by Lapid and Rogawski for $G L(2)$. Lapid and Rogawski obtain the descent criterion from a more general result, classifying cuspidal automorphic representations $\pi$ of $G L_{2}\left(\mathbf{A}_{K}\right)$ such that $\pi^{\sigma} \simeq \pi \otimes \omega$, for some automorphism $\sigma$ of $K$ and a Hecke character $\omega$ of $K$, and it is this more general result that is of crucial importance to us in obtaining a generalisation of the above results to the solvable case.

At first sight the general question seems to have a negative answer, since it is false even for characters. It is possible to construct Hecke characters of a number field $K$, invariant with respect to an abelian group of automorphisms of the field which do not descend:

Example. Let $H$ be a Heisenberg type group, i.e., a non-split central extension

$$
0 \rightarrow \mathbb{Z} / p \mathbb{Z} \rightarrow H \rightarrow \mathbb{Z} / p \mathbb{Z} \times \mathbb{Z} / p \mathbb{Z} \rightarrow 0
$$

Consider a field extension $M / k$ with Galois group isomorphic to $H$, and let $K$ be the field of invariants of $M$ by the centre of $H$. Let $\chi$ be the Hecke character of $K$, corresponding to the extension $M / K$ given by class field theory. Since the extension splits over any subgroup of $G(K / k)$ of order $p$, it follows that $\chi$ is invariant with respect to the subgroups generated by any element of $G(K / k)$, and hence is $G(K / k)$-invariant. However $\chi$ cannot descend to an idele class character of $k$, as this would imply that the extension $M / k$ is abelian, contrary to our choice of $H$.

Thus even for the simplest examples of non-cyclic groups and for $G L(1)$, we find that invariant automorphic forms do not descend in general. Twisting a cuspidal automorphic representation which lies in the image of base change, by an invariant character which does not descend, we have examples of invariant automorphic representations which do not descend.

However, contrary to the expectation that the nonabelian, higher rank analogue will in general be more difficult than the abelian case, we shall see below that the 'obstruction' to descent lies essentially at the level of characters. We show that invariant automorphic representations descend after twisting by an invariant character.

Theorem 1. Let $K / k$ be a solvable extension of number fields, and let $\Pi$ be a unitary, cuspidal automorphic representation of $G L_{2}\left(\mathbf{A}_{K}\right)$ which is $\operatorname{Gal}(K / k)$ invariant. Then there exists a $G(K / k)$-invariant Hecke character $\psi$ of $K$, and 
a cuspidal automorphic representation $\pi$ of $G L_{2}\left(\mathbf{A}_{K}\right)$ such that

$$
B C_{K / k}(\pi) \simeq \Pi \otimes \psi
$$

Further $\psi$ is unique upto base change to $K$ of a Hecke character of $k$.

Corollary 1. Suppose $K / k$ is a solvable extension with the property that invariant idele class characters of $K$ descend to $k$. Then any invariant, unitary, cuspidal automorphic representation of $G L_{2}\left(\mathbf{A}_{K}\right)$ lies in the image of the base change map $B C_{K / k}$.

In particular, we recover the classical formulation that invariant, unitary, cuspidal automorphic representations descend if $K / k$ is cyclic.

Remark 1. The proof depends on a theorem of Lapid and Rogawski (see [LR, Theorem 2]), classifying cuspidal automorphic representations $\pi$ of $G L_{2}\left(\mathbf{A}_{K}\right)$ such that $\pi^{\sigma} \simeq \pi \otimes \omega$, for some automorphism $\sigma$ of $K$ and a Hecke character $\omega$ of $K$. The work of Langlands was extended to $G L_{n}, n \geq 3$ by Arthur and Clozel $[\mathrm{AC}]$. It is to be expected that the Statement B of Lapid and Rogawski, generalizing [LR, Theorem 2] to arbitrary $n$, be valid in general. Granting this, our above theorem extends to $G L_{n}$, and we present the proof in the general case assuming Statement B of [LR].

Example. In the notation of the example given above, if $\Pi$ is a $G(K / k)$ invariant cuspidal automorphic representation of $G L_{n}\left(\mathbf{A}_{K}\right)$, then it descends to a cuspidal automorphic representation $\pi$ of an intermediate field extension, say $l$, of degree $p$ over $k$. If $\sigma$ denotes a generator of $G(l / k)$, then we have

$$
\pi^{\sigma} \simeq \pi \otimes \eta
$$

where $\eta$ satisfies, $\eta \circ N_{K / l}=1$, where for an extension of $E / F$ of number fields, $N_{E / F}$ denotes the norm map at the level of ideles. In this case $\eta$ is the base change of a Hecke character of $k$, and there exists a Hecke character $\psi$ of $l$ satisfying,

$$
\psi^{1-\sigma}=\eta .
$$

It can be checked that $\pi \otimes \psi$ is $G(l / k)$-invariant, and hence descends by the classical descent criterion.

We now characterize the fibres of the base change lift for solvable extensions of number fields.

Theorem 2. Let $K / k$ be a solvable extension of number fields. Let $\Pi$ be a cuspidal automorphic representation of $G L_{n}\left(\mathbf{A}_{K}\right)$. Suppose there exist cuspidal automorphic representations $\pi, \pi^{\prime}$ of $G L_{n}\left(\mathbf{A}_{k}\right)$ such that,

$$
B C_{K / k}(\pi)=B C_{K / k}\left(\pi^{\prime}\right)=\Pi .
$$

Then there exists a character $\chi$ of $C_{k}$ corresponding via class field theory to a character of $G(K / k)$, such that

$$
\pi^{\prime} \simeq \pi \otimes \chi
$$


Morever if $\chi$ is non-trivial, the representations $\pi$ and $\pi \otimes \chi$ are distinct.

We expect that the above theorems would further our understanding of the Artin conjecture for representations of the absolute Galois group of a number field into $G L_{n}(\mathbb{C})$ with solvable image. The proof of these theorems is by induction, using the descent criterion of Langlands, Arthur and Clozel for cyclic extensions of prime degree. We first prove Theorem 2, by induction and using the characterisation of automorphically induced representations by Labesse and Langlands in the case of $G L(2)$, and by Arthur and Clozel for general $G L(n)$ $[\mathrm{AC}]$.

The assumption that the character $\psi$ in Theorem 1 be invariant, is crucial in the proof of the descent property for the automorphically induced representations. It is not necessary to assume that $\psi$ be invariant, if the representation is not automorphically induced. To show descent, we use the invariance property of $\psi$, induction hypothesis and Theorem 2 , to reduce to a situation considered by Lapid and Rogawski. Using their results and the assumption that $\Pi$ is cuspidal, allows us to prove Theorem 1.

Remark 2. The motivation for these theorems come from a theorem of Tate (see $[\mathrm{Se}]$ ),

$$
H^{2}\left(G_{K}, \mathbb{C}^{*}\right)=1
$$

where $G_{K}$ denotes the absolute Galois group of $K$. Let $K / k$ be a Galois extension of number fields, not necessarily solvable. If $\rho$ is an irreducible, $G(K / k)$-invariant representation of the Galois group $G_{K}$ into $G L_{n}(\mathbb{C})$, then it can be extended as a projective representation of $G_{k}$ to $P G L(n, \mathbb{C})$. By Tate's theorem, this projective representation has a lift to $G L(n, \mathbb{C})$. Restricting back to $G_{K}$, it can be checked that the lift differs from the original representation by an invariant character. In this regard, we refer also to a theorem of Labesse [Lab1], showing in essence the analogue of Tate's theorem for admissible homomorphisms of the Weil group.

Remark 3. An elliptic curve $E$ over a Galois extension $K$ of $\mathbb{Q}$ is said to be a $\mathbb{Q}$-curve, if it is isogenous to all it's conjugates by elements of $G(K / \mathbb{Q})$. It has been shown by Elkies and Ribet [Ri], that a non CM, $\mathbb{Q}$-elliptic curve is defined upto isogeny over a $(2,2, \cdots, 2)$-extension $L$ over $\mathbb{Q}$. If we assume a generalized version of the Shimura-Taniyama-Weil conjecture, then we obtain an automorphic representation $\Pi$ of $G L\left(2, \mathbf{A}_{K}\right)$, such that the associated $L$ functions are the same. It follows that $\pi$ is $G(L / \mathbb{Q})$-invariant. By our Theorem, $\Pi$ descends to an automorphic representation $\pi$ of $G L\left(2, \mathbf{A}_{\mathbb{Q}}\right)$ after twisting by an invariant Hecke character of $L$. $\pi$ will give raise to a holomorphic form and an associated abelian variety.

Notation. $K / k$ is a solvable Galois extension of number fields. Where the proofs are by induction on the order of the Galois group, we assume we have a filtration of Galois extensions,

$$
K=K_{0} \supset K_{1} \supset K_{2}=k,
$$


where either $K_{1} / K_{2}$ or $K_{0} / K_{1}$ is a cyclic extension of prime degree. The subscripts for the representations correspond to the subscripts of the associated fields. The automorphic representations considered are unitary upto twisting by a character. For a number field $K, C_{K}$ will denote the idele class group of $K$.

\section{Fibres of the base change lift}

We first begin with the proof of Theorem 2, since it is needed for the proof of Theorem 1 .

Proof of Theorem 2. We argue by induction. Assuming the hypothesis of the theorem, we obtain by Langlands ([L]), Arthur and Clozel [AC], that if $K / k$ is a cyclic extension of prime degree $p$, then there exists a character $\chi$ such that $\pi^{\prime} \simeq \pi \otimes \chi$. In this case, we only need to check the last statement of the theorem: if $\pi \simeq \pi \otimes \chi$, and $\chi$ is a non-trivial character of $C_{k}$ corresponding to $K$, then $\pi$ is necessarily automorphically induced from a representation of $G L_{n / p}\left(\mathbf{A}_{K}\right)$, by the characterisation of automorphically induced representations by LabesseLanglands [LL] and Arthur-Clozel[AC]. It follows that $\Pi=B C_{K / k}(\pi)$ is noncuspidal, contradicting our assumption on $\Pi$. Thus the theorem is true for cyclic extensions of prime degree.

Since $K / k$ is solvable, we can assume by induction that we have a filtration

$$
K=K_{0} \supset K_{1} \supset K_{2}=k,
$$

where $K_{1} / K_{2}$ is a cyclic extension of prime degree, and the theorem is true for $K_{0} / K_{1}$. that

Suppose $\pi_{2}, \pi_{2}^{\prime}$ are cuspidal automorphic representations of $G L_{n}\left(\mathbf{A}_{K_{2}}\right)$ such

$$
B C_{K_{0} / K_{2}}\left(\pi_{2}\right) \simeq B C_{K_{0} / K_{2}}\left(\pi_{2}^{\prime}\right) \simeq \Pi
$$

Let $B C_{K_{1} / K_{2}}\left(\pi_{2}\right)=\pi_{1}$ and $B C_{K_{1} / K_{2}}\left(\pi_{2}^{\prime}\right)=\pi_{1}^{\prime}$.

By induction hypothesis, $\pi_{1}^{\prime} \simeq \pi_{1} \otimes \chi_{1}$, for some idele class character $\chi_{1}$ of $C_{K_{1}}$ corresponding via class field theory to a character of the Galois group $G\left(K_{0} / K_{1}\right)$. Let $\sigma$ be a generator of $G\left(K_{1} / K_{2}\right)$. We have

$$
\begin{gathered}
\pi_{1} \otimes \chi_{1} \simeq \pi_{1}^{\prime} \simeq \pi_{1}^{\prime \sigma} \simeq \pi_{1}^{\sigma} \otimes \chi_{1}^{\sigma} \simeq \pi_{1} \otimes \chi_{1}^{\sigma} . \\
\text { Hence } \quad \pi_{1} \simeq \pi_{1} \otimes \chi_{1}^{1-\sigma} .
\end{gathered}
$$

If $\chi_{1} \neq \chi_{1}^{\sigma}$, let $f$ denote the order of $\chi_{1}^{1-\sigma}, p$ a prime dividing $f$, and let

$$
\nu=\chi_{1}^{(1-\sigma) f / p}
$$

$\nu$ is a non-trivial character of $G\left(K_{0} / K_{1}\right)$ of order $p$ satisfying,

$$
\pi_{1} \simeq \pi_{1} \otimes \nu
$$

By the characterisation of automorphically induced representations due to Labesse and Langlands [LL] for $G L(2)$, and by Arthur and Clozel [AC] for $G L(n)$, it follows that $\pi_{1}$ is automorphically induced from a Hecke character 
$\theta$ belonging to the class field $K_{\nu}$ defined by $\nu$. But $K_{\nu} \subset K_{0}$, and it follows that $\Pi$ is not cuspidal, contrary to our assumption on $\Pi$. Hence we have that $\chi_{1}$ is invariant,

$$
\chi_{1}=\chi_{1}^{\sigma} .
$$

Since $K_{1} / K_{2}$ is cyclic of prime degree, it follows that $\chi_{1}$ extends to a character $\chi_{2}$ of $G\left(K_{0} / K_{2}\right)$ (or at the level of ideles, $\left.\chi_{1}=\chi_{2} \circ N_{K_{1} / K_{2}}\right)$. Then

$$
B C_{K_{1} / K_{2}}\left(\pi_{2} \otimes \chi_{2}\right) \simeq \pi_{1} \otimes \chi_{1} \simeq \pi_{1}^{\prime} \simeq B C_{K_{1} / K_{2}}\left(\pi_{2}^{\prime}\right) .
$$

Hence we have a Hecke character $\theta$ corresponding to a character of $G\left(K_{1} / K_{2}\right)$, such that

$$
\pi_{2}^{\prime} \simeq \pi_{2} \otimes \chi_{2} \theta
$$

and $\chi_{2} \theta$ defines a character of $G\left(K_{0} / K_{2}\right)$.

It is clear that the representations $\pi_{2}$ and $\pi_{2} \otimes \chi$ are distinct for a character $\chi$ of $G\left(K_{0} / K_{2}\right)$, even when $\pi_{2}$ is automorphically induced from a character of a field $M_{2}$ over $K_{2}$, since it follows from the cuspidality of $\Pi$ that the fields $M_{2}$ and $K_{0}$ are disjoint.

\section{Descent for invariant representations}

Before we proceed to the proof of Theorem 1, we prove a preliminary lemma, which also proves the uniqueness assertion about $\psi$ in Theorem 1.

Lemma 1. Let $K / k$ be a solvable extension, and $\Pi$ be a cuspidal automorphic representation of $G L_{n}\left(\mathbf{A}_{K}\right)$. Suppose $\chi$ is a $G(K / k)$ invariant Hecke character of $K$, such that both $\Pi$ and $\Pi \otimes \chi$ are in the image of base change from $k$. Then $\chi$ lies in the image of base change.

Proof. By induction. The theorem is true for cyclic extensions of prime degree. Assume we have $K=K_{0} \supset K_{1} \supset K_{2}=k$ with $K_{0} / K_{1}$ prime of degree $p$. Since $\chi$ is invariant, $\chi=\chi_{1} \circ N_{K_{0} / K_{1}}$ for some idele class character $\chi_{1}$ of $C_{K_{1}}$. Suppose $\pi_{1}, \pi_{1}^{\prime}$ are cuspidal automorphic representations of $G L_{n}\left(\mathbf{A}_{K_{1}}\right)$ are cuspidal automorphic representations of $G L_{n}\left(\mathbf{A}_{K_{1}}\right)$ lying in the image of base change from $K_{2}$ to $K_{1}$, such that

$$
B C_{K_{0} / K_{1}}\left(\pi_{1}\right) \simeq \Pi \text { and } B C_{K_{0} / K_{1}}\left(\pi_{1}^{\prime}\right)=\Pi \otimes \chi .
$$

Now both $\pi_{1}^{\prime}$ and $\pi_{1} \otimes \chi_{1}$ base change to $\Pi \otimes \chi$. By the description of the fibres of base change for a cyclic extension of prime degree, we obtain,

$$
\pi_{1}^{\prime} \simeq \pi_{1} \otimes \chi_{1} \eta_{1}
$$

for some Hecke character $\eta_{1}$ of $K_{1}$ corresponding to the extension $K_{0} / K_{1}$. By hypothesis both $\pi_{1}$ and $\pi_{1}^{\prime}$ lie in the image of base change from $K_{2}$ to $K_{1}$, and we will be through by induction, if we can only show that $\chi_{1} \eta_{1}$ is $G\left(K_{1} / K_{2}\right)$ invariant, i.e., for any $\sigma \in G\left(K_{1} / K_{2}\right)$ we have to show that

$$
\left(\chi_{1} \eta_{1}\right)^{\sigma}=\chi_{1} \eta_{1} \text {. }
$$


Since $\pi_{1}^{\prime}$ and $\pi_{1}$ are $\sigma$-invariant, we have

$$
\pi_{1} \otimes \chi_{1} \eta_{1} \simeq \pi_{1}^{\prime} \simeq \pi_{1}^{\prime \sigma} \simeq \pi_{1} \otimes\left(\chi_{1} \eta_{1}\right)^{\sigma} .
$$

Hence we obtain

$$
\left(\chi_{1} \eta_{1}\right)^{1-\sigma}=\nu
$$

where $\nu$ satisfies

$$
\pi_{1} \otimes \nu \simeq \pi_{1} .
$$

Since $\chi$ is $G\left(K_{0} / K_{2}\right)$-invariant, we have

$$
\chi_{1}^{\sigma}=\chi_{1} \eta_{1}^{i} \quad \text { and } \quad \eta_{1}^{\sigma}=\eta_{1}^{j},
$$

for some integers $i, j$. Hence,

$$
\nu=\left(\chi_{1} \eta_{1}\right)^{1-\sigma}=\eta_{1}^{l},
$$

for some integer $l$. But by the criterion of Labesse-Langlands for $G L(2)$ and Arthur-Clozel for general $G L(n)$, we see that $\Pi$ is not cuspidal, contradicting our hypothesis that $\Pi$ is cuspidal.

Remark 4. Suppose $K / k$ and $L / k$ are disjoint quadratic extensions of $k$. Let $\pi$ be a cuspidal representation of $k$ induced from a Hecke character of $L$, and let $\Pi$ be the base change of $\pi$ to $K$. Let $\nu$ be the Hecke character of $K$ corresponding to the extension $K L / K . \quad \nu$ restricted to $C_{k}$ is trivial, and hence there is a Hecke character $\psi$ of $K$ satisfying $\psi^{1-\sigma}=\nu$, where $\sigma$ denotes the non-trivial automorphism of $K / k$. Since $\Pi$ satisfies $\Pi \otimes \nu=\Pi$, we obtain that $\Pi \otimes \psi$ is $\sigma$-invariant. Thus both $\Pi$ and $\Pi \otimes \psi$ lie in the image of base change from $k$, but $\psi$ is not a base change character.

If $\Pi$ is not automorphically induced, then it is not necessary to require that the character $\chi$ be invariant. In this case, if both $\Pi$ and $\Pi \otimes \chi$ are in the image of base change from $k$, then $\chi$ is invariant and lies in the image of base change.

Proof of Theorem 1. We assume there is a sequence of fields

$$
K=K_{0} \supset K_{1} \supset K_{2}=k,
$$

where $K_{1} / K_{2}$ is a cyclic extension of prime degree $p$, and the theorem is true for $K_{0} / K_{1}$, i.e., there exists a $G\left(K_{0} / K_{1}\right)$-invariant Hecke character $\psi_{0}$ of $K_{0}$ such that

$$
\Pi \otimes \psi_{0}=B C_{K_{0} / K_{1}}\left(\pi_{1}\right),
$$

where $\pi_{1}$ is a cuspidal automorphic representation of $G L_{n}\left(\mathbf{A}_{K_{1}}\right)$. Let $\sigma$ be a generator of $G\left(K_{1} / K_{2}\right)$, and let $\tau$ be an element of $G\left(K_{0} / K_{2}\right)$ lifting $\sigma$. Then

$$
B C_{K_{0} / K_{1}}\left(\pi_{1}^{\sigma}\right) \simeq \Pi^{\tau} \otimes \psi_{0}^{\tau} \simeq \Pi \otimes \psi_{0}^{\tau} \simeq\left(\Pi \otimes \psi_{0}\right) \otimes \psi_{0}^{-1+\tau} .
$$


Now $\psi_{0}^{-1+\tau}$ is $G\left(K_{0} / K_{1}\right)$-invariant. By Lemma 1 , since both $\Pi \otimes \psi_{0}$ and $(\Pi \otimes$ $\left.\psi_{0}\right) \otimes \psi_{0}^{-1+\tau}$ lie in the image of base change, it follows that there exists an idele class character $\chi_{1}$ such that

$$
\psi_{0}^{-1+\tau}=\chi_{1} \circ N_{K_{0} / K_{1}}
$$

and hence $B C_{K_{0} / K_{1}}\left(\pi_{1}^{\sigma} \otimes \chi_{1}^{-1}\right) \simeq \Pi \otimes \psi_{0}$.

By Theorem 2, characterising the fibres of the base change lift, we conclude that there is an idele class character $\chi_{1}^{\prime}$ corresponding via class field theory to a character of $G\left(K_{0} / K_{1}\right)$, such that

$$
\pi_{1}^{\sigma} \simeq \pi_{1} \otimes \chi_{1}^{\prime} \otimes \chi_{1}=\pi_{1} \otimes \eta_{1},
$$

where $\eta_{1}=\chi_{1}^{\prime} \chi_{1}$. We have

$$
\eta_{1} \circ N_{K_{0} / K_{1}}=\psi_{0}^{-1+\tau},
$$

where $\psi_{0}$ is $G\left(K_{0} / K_{1}\right)$-invariant.

Our aim now is to prove that $\eta_{1}$ restricted to $C_{K_{2}}$ is trivial. Let $\eta_{2}=\eta_{1} \mid C_{K_{2}}$. We have,

$$
\begin{aligned}
\eta_{2} \circ N_{K_{0} / K_{2}} & =\eta_{2} \circ N_{K_{1} / K_{2}} \circ N_{K_{0} / K_{1}} \\
& =\eta_{1}^{1+\sigma+\cdots+\sigma^{p-1}} \circ N_{K_{0} / K_{1}} \\
& =\left(\eta_{1} \circ N_{K_{0} / K_{1}}\right)^{1+\tau+\cdots+\tau^{p-1}} \\
& =\left(\psi_{0}^{-1+\tau}\right)^{1+\tau+\cdots+\tau^{p-1}} \quad \text { by } 3.1 \\
& =\psi_{0}^{-1+\tau^{p}} \\
& =1
\end{aligned}
$$

Here the last line follows since $\psi_{0}$ is $G\left(K_{0} / K_{1}\right)$-invariant, and from the fact that $\tau^{p} \in G\left(K_{0} / K_{1}\right)$, since $\sigma$ is assumed to be of order $p$.

If $\eta_{2}$ is not trivial, then $\eta_{2}$ is a Dirichlet character of order $l$ dividing $n$. Let $M_{2}$ be the extension of $K_{2}$ corresponding to $\eta_{2}$ by class field theory. We have $M_{2} \cap K_{1}=K_{2}$, and let $M_{1}$ be the compositum of $K_{1}$ and $M_{2}$. By Theorem $2 \mathrm{~b}$ and Statement B.b) of Lapid and Rogawski [LR], we conclude that

$$
\pi_{1}=A I_{M_{1} / K_{1}}\left(B C_{M_{1} / M_{2}}\left(\pi_{M_{2}}\right) \otimes \theta\right),
$$

where $\pi_{M_{2}}$ is a cuspidal automorphic representation of $G L\left(n / l, \mathbf{A}_{M_{2}}\right)$ and $\theta$ is a Hecke character of $M_{1}$ satisfying

$$
\theta^{\sigma^{\prime}-1}=\eta_{1} \circ N_{M_{1} / K_{1}}
$$

where $\sigma^{\prime}$ is a lift of $\sigma$ to the extension $M_{2} / K_{2}$. (The notation $A I_{M_{1} / K_{1}}$ stands for automorphic induction for the extension $\left.M_{1} / K_{1}\right)$. In particular, we see that $\pi_{1}$ is automorphically induced from the field $M_{1}$. But the above calculation implies that the field $M_{2} \subset K_{0}$, and this implies that $\Pi$ is not cuspidal, contrary to our assumption. We remark here that the theorem of Lapid and Rogawski has been proved upon the assumption that a relevant fundamental lemma holds. It is known that this lemma holds for $G L(2)$. 
Hence $\eta_{1} \mid C_{K_{2}}$ is trivial. We can then find a character $\alpha$ satisfying

$$
\alpha^{1-\sigma}=\eta_{1}
$$

We check that $\pi_{1} \otimes \alpha$ is $G\left(K_{1} / K_{2}\right)$-invariant,

$$
\begin{aligned}
\left(\pi_{1} \otimes \alpha\right)^{\sigma} & =\pi_{1}^{\sigma} \otimes \alpha \eta_{1}^{-1} \quad \text { by } 3.2 \\
& =\pi_{1} \otimes \alpha .
\end{aligned}
$$

Hence $\pi_{1} \otimes \alpha$ descends to $K_{2}$. Hence we obtain that $\Pi \otimes \psi_{0} \otimes\left(\alpha \circ N_{K_{0} / K_{1}}\right)$ descends.

To finish the proof, we have to check that $\psi_{0} \otimes\left(\alpha \circ N_{K_{0} / K_{1}}\right)$ is $G\left(K_{0} / K_{2}\right)$ invariant, and for this it is enough to check that $\psi_{0} \otimes\left(\alpha \circ N_{K_{0} / K_{1}}\right)$ is $\tau$-invariant:

$$
\begin{aligned}
\left(\psi_{0} \otimes\left(\alpha \circ N_{K_{0} / K_{1}}\right)\right)^{\tau} & =\psi_{0}^{\tau} \otimes\left(\alpha \circ N_{K_{0} / K_{1}}\right)\left(\eta_{1} \circ N_{K_{0} / K_{1}}\right)^{-1} \quad \text { by } 3.2 \\
& =\psi_{0} \otimes\left(\alpha \circ N_{K_{0} / K_{1}}\right) \quad \text { by } 3.1 .
\end{aligned}
$$

Remark 5. In view of Theorem 1 and the descent property for irreducible invariant representations of the Galois group, it it tempting to consider that the descent criterion would work better for $S L_{n}$ rather than $G L_{n}$. However it is false that invariant automorphic representations (or $L$-packets) of $S L_{n}\left(\mathbf{A}_{K}\right)$ descend by a cyclic automorphism $\sigma$ of prime degree of $K$. For instance consider a cuspidal automorphic representation on $G L_{2}\left(\mathbf{A}_{K}\right)$ satisfying $\pi^{\sigma} \simeq \pi \otimes \eta$ such that $\eta_{k}:=\eta \mid C_{k}$ is non-trivial, where $k=K^{\sigma}$. The corresponding $L$-packet defined by the restriction of $\pi$ to $S L_{2}\left(\mathbf{A}_{K}\right)$ is invariant by $\sigma$. If it were true, that this $L$-packet descends, then it would amount to saying that $\pi$ descends after twisting by a character $\psi$ which is not necessarily invariant. This amounts to saying that $\pi \simeq \pi \otimes \eta \psi^{\sigma-1}$. By the theorem of Lapid and Rogawski, we obtain that $\eta=\psi^{1-\sigma}\left(\eta_{k} \circ N_{K / K}\right)$. But the right hand side character restricts trivially to $C_{k}$, and this contradicts the assumption that $\eta_{k}$ is non-trivial. However in the 'general case' when the representations are not induced, it may prove to be better to do base change for $S L_{n}\left(\left({ }^{L} S L_{n}\right)^{0}=P G L_{n}\right)$, rather than $G L_{n}$, in order to remove the ambiguity of twisting by a character to make invariant representations descend.

\section{Acknowledgement}

I am indebted to D. Prasad for stimulating my interest in this question, and for pointing out a gap in an earlier version of this paper.

\section{References}

[AC] J. Arthur, and L. Clozel, Simple algebras, base change, and the advanced theory of the trace formula, Annals of Mathematics Studies, 120. Princeton University Press, Princeton, NJ, 1989.

[Lab] J.-P. Labesse, Noninvariant base change identities, Mém. Soc. Math. France (N.S.) No. 61, (1995). 
[Lab1] _ Cohomologie, L-groupes et fonctorialité, Compositio Math. 55 (1985), 163184.

[LL] J.-P. Labesse and R. Langlands, L-indistinguishablity for SL(2), Canad. J. Math. 31 (1979), 726-785.

[L] R. Langlands, Base change for GL(2), Annals of Mathematics Studies, 96. Princeton University Press, Princeton, NJ, 1980.

[LR] E. Lapid and J. Rogawski, On twists of cuspidal representations of GL(2), Forum Math. 10 (1998), 175-197.

[R] C.S. Rajan, On the image and the fibres of the non-normal cubic lift, C. R. Math. Acad. Sci. Soc. R. Can. 22 (2000), 1-6.

[Ri] K. Ribet, Fields of definition of abelian varieties with real multiplication, Arithmetic geometry (Tempe, AZ, 1993), 107-118, Contemp. Math., 174, Amer. Math. Soc., Providence, RI, 1994.

[Se] J.-P. Serre, Modular forms of weight one and Galois representations, Algebraic number fields: $L$-functions and Galois properties (Proc. Sympos., Univ. Durham, Durham, 1975), pp. 193-268. Academic Press, London, 1977.

Tata Institute of Fundamental Research, Homi Bhabha Road, Bombay - 400005 , INDIA.

E-mail address: rajan@math.tifr.res.in 